\title{
Tecniche di ripetizione nella "conversazione" vittoriniana
}

\section{John Campana}

La ripetizione è una tecnica del linguaggio molto diffusa nella civiltà classica occidentale dove, fra l'altro, venne usata retoricamente per amplificare, conferire maggiore enfasi al discorso ed intensificare il valore della parola, frase o concetto ripetuto. ${ }^{1}$ Altrettanto frequente nella piú antica civiltà orientale, la ripetizione fu adoperata come mezzo di approfondimento della coscienza, tutt'ora operante nel concetto indú detto "mantra."2

Nel suo uso euristico della ripetizione, Vittorini si avvicina di piú alla tradizione orientale, data la sua insistenza sulla ricerca della verità nelle sue opere, come ci rivela ripetutamente la sua prefazione alla prima edizione del Garofano rosso del $1948 .{ }^{3}$ Senza dubbio Vittorini non aveva nessuna conoscenza dei testi filosofici indiani e orientali. L'importanza è che egli abbia seguito, seppure inconsciamente ed indipendentemente, un analogo schema narrativo atto a delineare l'itinerario "spirituale" del suo personaggio.

Generalmente, a parte qualche breve accenno, la critica ha trascurato, o ritenuto trascurabile, non solo questa tecnica, ma anche la sua frequenza nelle opere di Vittorini. Alcuni hanno definito ossessiva o ridondante la ripetizione vittoriniana. Altri l'hanno denominata ecolalia considerandola perfino indice di povertà linguistica. Altri ancora, però, hanno intuito la suggestività poetica del non detto tra una ripetizione e l'altra ed anche il carattere seducente dell'allucinata e allucinante concisione della pagina vittoriniana. ${ }^{4}$

In questo studio, sarà nostra intenzione analizzare e determinare il ruolo preciso della ripetizione nel progresso della coscienza del protagonista di Conversazione in Sicilia (CS) nel suo "viaggioconversazione."

Nel descrivere l'itinerario dell'io di Silvestro, critici come Bernardi, Pautasso, David, si sono serviti di modelli strutturali antropologici, strutturalistici o psicoanalitici; tuttavia le loro consi- 
derazioni si sono concentrate piú sugli aspetti esteriori del viaggio che sul divenire interiore del protagonista. Il viaggio di Silvestro non è semplicemente ritorno mitico alla madre, all'isola o all'infanzia; è epopea alla ricerca dell'io, di verità, di umanità e di significato. Inoltre, questo viaggio interiore-verticale al "cuore puro della Sicilia," ha tutte le caratteristiche di una vera e propria ascesi mistica, essendo anche simbolicamente l'odissea dalla morte alla vita, dall'oblio alla memoria, dal simbolo all'archetipo, dall'astratto al concreto.

Piú che una semplice figura stilistica, la ripetizione, nell'uso programmatico che ne fa Vittorini, genera una forza o energia evocatrice nelle parole o immagini, facendo risuonare di un significato ricco ed allusivo il silenzio fra una ripetizione e l'altra. Altrimenti detto, la ripetizione sta al "corpo" del romanzo come il "mantra" sta ai "chakra" del corpo umano tesc alla meditazione e alla scoperta di alte verità e di realtà profonde. Anche CS si prefigge questi scopi concentrandosi e ripiegandosi, alle volte ossessivamente, data l'urgenza del suo messaggio, sui grandi temi della meditazione personale di Vittorini, cioè, quelli della verità (basta osservare la frequente ripetizione della parola verità nella prefazione al Garofano rosso), e del "genere umano offeso," ai quali nel suo viaggio-conversazione il protagonista cerca di trovare una risposta.

Seguendo lo schema dei "chakra" delineato nelle note, si potrebbe affermare che anche CS, nella struttura esterna del viaggio orizzontale di Silvestro, sia composto di diversi "chakra," i quali rispecchiano il dinamismo interno del viaggio verticale e della progressione della coscienza del protagonista.

La ripetizione assume diversi aspetti in CS. Ci sono casi di ripetizione a distanza, tramite cui una parola, un' immagine o persino un personaggio acquista il valore di un "leitmotiv" o di un simbolo. Se si traccia l'intinerario del colore nero nei romanzi vittoriniani, si arriverà a confermare il valore universale di negatività insito nel simbolismo universale della parola nero. Per quanto riguarda la poetica di Vittorini, il nero è il distintivo del "non uomo," come pure lo è il colore melanzana che serve a definire i cappelli degli impiegati in Erica e $i$ suoi fratelli, e il cappotto di Senza Baffi in CS. La ripetizione di questi ed altri colori trasmette ai lettori un messaggio velato, un'espressione del non detto del carattere dei personaggi a cui questi colori vengono attribuiti. Allo stesso modo, il rosso della bandierina appesa al piffero di Muso-di-fumo e il rosso del drappo del panniere Porfirio, diventa una parola spia, tramite la ripetizione, di una piú 
che possibile allusione politica di Vittorini che si riallaccia al tema generale del "genere umano offeso."

La ripetizione è il modo che Vittorini presceglie per "dichiarare senza dire," come spiega nella sua prefazione al Garofano rosso, il modo per ricordarci che si scrive perché si ha una verità da dire: "e se torno a scrivere non è perché mi accorga di 'altre' verità che si possono aggiungere, e dire 'in piú,' dire 'inoltre,' ma perché qualcosa che continua a mutare nella verità mi sembra esigere che non si smetta mai di ricominciare a dirla." Sembrerebbe che, dato il carattere unico della verità, sia impossibile qualsiasi tipo di ripetizione. Tuttavia, per dirla con Kavin, quando si scrive della verità la si può osservare da diverse prospettive. Il cambiare i termini serve ad amplificare la realtà rendendola piú stabile e piú generale. ${ }^{5}$

Nello stesso modo in cui i colori ripetuti a distanza hanno rafforzato il concetto di "non uomo" nella tematica vittoriniana, le parole-simbolo "nonno" e "cinese," tramite lo stesso dinamismo della ripetizione, allargano la sfera semantica del concetto vittoriniano di "piú uomo" e del tema del "genere umano offeso," il quale, attraverso la ripetizione, diventa quello che Forester chiama "expanding symbol" ovvero "simbolo in espansione." 6

Molti altri sono gli esempi di ripetizione a distanza che generano nuovi significati raggiungendo sfere di realtà superiore o "maggiore," come la definisce Vittorini. Tra gli esempi piú salienti ci sono quelli delle parole-simbolo "acqua" e "piffero," che abbiamo già esaminato altrove dal punto di vista di sinestesia e di riduzione al significato essenziale. ${ }^{7}$ Le immagini liquide in CS (pioggia, mare, lacrime, miele, sangue, sudore, vino, ecc.) si riducono alla "realtà maggiore" e simbolica di "acqua viva," e le immagini sonore (le parole stesse della "conversazione" in Sicilia, la lettera del padre, i giornali squillanti, il lamento, le zampogne, i campanelli, i vecchi motivi ecc.) vengono a confluire nell'unico suono-simbolo (vera e propria "mantra" di CS) della esclamazione ipersemantica e meta-linguistica "EHM!" pronunciata nel cimitero dal fratello morto di Silvestro.

In CS si possono anche isolare centri principali che corrispondono ai vari stadi del viaggio di Silvestro da Milano alla Sicilia. Queste tappe, collegate in parte dalle varie ripetizioni a distanza menzionate brevemente sopra, acquistano inoltre una vera e propria autonomia per virtú dell'uso particolare della ripetizione a contatto nelle ultime tappe del viaggio. La ripetizione, in questi 
stadi finali della progressione della coscienza, diventa piú frequente, finché i vari "chakra" del romanzo si ricollegano, essendo l'uno la modulazione tematica e poetica dell'altro. Le varie tappe del viaggio, nell'ordine lineare e verticale del progresso di Silvestro si possono dividere nel modo seguente: alienazione (lo stato di inerzia in preda agli "astratti furori"), tentativo di inserimento (sul traghetto e nel mondo della miseria), cognizione del male e del dolore (sul treno e negli inferi del "genere umano operaio"), rigenerazione (fisica e simbolica, e la parola "uomo"), la quarta dimensione, il regno dell'illusione, e finalmente l'ascesi della coscienza o la reintegrazione.

"Astratti furori," motivo centrale del primo capitolo di CS diventa anche, tramite la ripetizione, "simbolo in espansione" come il tema del "genere umano perduto" a cui dà l'avvio. Benché nominati una sola volta, gli "astratti furori" ossessionano il primo capitolo con la loro presenza spettrale resa tale dalla ripetuta insistenza sulla particella negativa "non" che viene strategicamente fatta precedere agli aggettivi "eroici" e "vivi" e le frasi "aver febbre di" e "aver voglia di." Il "non," che fra l'altro rappresenta l'apatia e l'indifferenza del protagonista, attinge il culmine della disperazione e della negatività quando si abbina al sostantivo "speranza." In seguito, il "non" si modula diventando "né mai," "nulla," "nessuna," il che mette in rilievo il vuoto esistenziale di Silvestro, il quale non esita a confessarci che si sentiva come se non avesse mai avuto un giorno di vita. Non vita, dunque, la sua, e anche non speranza, come un "sordo sogno" che non riesce neppure a diventare incubo. Energia negativa quella emanata dagli astratti furori, che si insinua perfino nelle cose e negli eventi rendendoli altrettanto confusi e amorfi. Le parole generano nebbia e ne vengono assorbite. Non parola, dunque, silenzio, e soprattutto non signficato, fabbricazione della parola ("tipografo-linotipista") e il vano tentativo di trovare un senso nelle parole stampate ("sfogliare il dizionario").

Con la lettera del padre, primo barlume di significato in forma di parole stampate, entra in gioco la particella reiterativa "ri," che segna l'inizio del risveglio della coscienza, della penetrazione di una realtà che sarà ricordata, riveduta, riconosciuta, rivissuta, e finalmente riavuta ("riavere la mia infanzia"). Entusiasmato dalla possibilità di intravedere un segno di vita nella lettera, Silvestro riprende la lettera, la rilegge e vi riconosce se stesso bambino. Il sipario della memoria, apertosi solo per un istante, si richiude, e le immagini di vita destategli dalla lettera ritornano allo stato sfocato e amorfo nella nebbia degli astratti furori, e 
Silvestro ridiventa triste fantasma della indifferenza privo d'identità: "mi era lo stesso," dice, "era lo stesso." Queste frasi non sono altro che la ripetizione dello stesso "sordo sogno" e la stessa non speranza di prima.

La lettera, tuttavia, gli aveva piantato dentro un seme di significato che egli rimetterà a fuoco durante il suo viaggio, ed anche una briciola di speranza di poter forse popolare il vuoto di "qualcosa che poteva anche non essere una cosí scura quiete e una cosí sorda non speranza" (CS, p. 575). E cosí intraprende il viaggio in Sicilia, un viaggio interno, verticale, nell'imo del proprio essere dove verranno a confluire tempo e memoria aprendogli il varco alla "quarta dimensione." La lettera inoltre, serve da netto contrasto all'universo di significazione negativa (non, mai, nulla) o di significato latente, inerte (dizionario), innalzando momentaneamente il sordo sogno dal subconscio alla superficie della cognizione e della memoria.

Con le parole "bambino," "ragazzo" e "infanzia" si crea, poi, un moto regressivo nel tempo, e uno slancio progressivo nella memoria, lontano dall'oblio partorito dagli astratti furori. L'idea di tempo e memoria sarà rievocata piú tardi durante il viaggio dall' insistenza sulle parole "vecchio" e "antico" ripetute sulla soglia della quarta dimensione. Nel frattempo Silvestro risolve di tornare al luogo delle sue origini per poter stabilire un contatto con se stesso, con le cose del mondo e con il "genere umano perduto." Solo cosí può arrivare a riconoscere che anche lui fa parte dell' "astrazione delle folle massacrate," e che gli astratti furori non sono altro che "le offese recate al mondo," non potenze negative che immobilizzano il senso della verità e della realtà in lui. Soltanto cosí può anche disfarsi dell'astrazione del suo essere alienato.

Il riconoscimento è graduale all' inizio del suo viaggio ed è affidato solamente a stimoli esterni. Silvestro riconosce il viaggio, le sue fughe da casa, e i vari nomi che popolano il suo attuale non essere. Amantéa, Maratéa e Gioia Tauro, sono per lui "nomi da sogni antichi," come pure lo è il sapore del formaggio che mangia sul traghetto. I nomi richiamano l'immagine del sordo sogno iniziale da cui provengono. Riecheggianti nella memoria inconscia di Silvestro, i nomi hanno il valore evocativo di un "mantra" se si considerano come maggiore messa a fuoco dell" evanescente sordo sogno.

L'interiorità del viaggio viene piú volte ribadita dalla ripetizione di certe parole spia come "punto" ("punto suo della terra," 
"punto della memoria") e dalle frasi "in me," "entro di me," che nello spazio delle cinque pagine dei primi tre capitoli vengono ripetute nove volte, miranti a localizzare la vera meta del viaggio interno che, tutto sommato, si trova nel protagonista stesso e viene chiamato "il cuore puro della Sicilia."

L'attività onirica dei sogni antichi viene ripresa sul traghetto, spostandosi questa volta, su un piano intermedio tra conscio e subconscio. Nel dormiveglia Silvestro riesce a captare, in modo meno evasivo, le immagini scarnificate della sua astrazione. Il dinamismo sinestetico dei suoi pensieri illustra i primi tentativi del protagonista nel "riavere" la sua infanzia: "Cosí un topo, d'un tratto, non era piú topo in me, era odore, sapore, cielo e il piffero suonava un attimo melodioso, non piú lamentoso" (CS, p. 576).

Ad ispirargli entusiasmo non sono soltanto i nomi antichi della Magna Grecia ma anche il sapore del formaggio che gusta sul traghetto. Gli astratti furori che prima si erano parzialmente materializzati nell'espressione "giornali squillanti" ed anche nelle frasi "genere umano perduto" e "topi scuri dei miei anni," continuano ad esteriorizzarsi in quel sapore antico (come i nomi) del formaggio. Le esperienze sensoriali del pellegrino gli rendono piú accessibile la soglia della quarta dimensione.

Per arrestare il flusso continuo della realtà di quel formaggio, Silvestro ripete sei volte la frase "Non c'è formaggio come il nostro." Questo lo fa anche per accomunarsi (tramite il possessivo "nostro") ai piccoli siciliani da cui si sentiva alienato, e per trovare conferma della nuova realtà/verità da lui scoperta per poi condividerla con loro. Per ora il suo tentativo d'inserimento resta vano. Piú tardi, durante il giro delle iniezioni, un'altra frase, questa volta pronunciata dalla madre ("Ho con me mio figlio"), servirà ad inserirlo ritualisticamente nella comunità del "genere umano affamato."

Nel formaggio, come nei nomi antichi, Silvestro sta pregustando la quarta dimensione nella quale verrà introdotto anche tramite i pasti, come quelli della sua infanzia, che riassaggia a casa della madre, Concezione. Nel ripetere la frase ai piccoli siciliani egli cerca di intrattenere il sentimento di una nuova speranza e di prolungare l'incanto procuratogli dalla nuova esperienza sensoriale. La ripetizione per Silvestro, allora, diventa tecnica del possedere nel procedimento interno di ricupero della sua infanzia. Ripetere, dunque, significa anche rivivere, ristabilire il contatto con l'originaria essenza delle cose e delle persone. 
La nota dell'antichità, fatta risuonare per la prima volta dall'accenno fatto ai nomi da sogni antichi e al sapore del formaggio, si carica di maggior valore simbolico tramite la ripetizione dell'aggettivo "antico" che, nella maggior parte dei casi, qualifica la parola "uomo." Il Gran Lombardo, prima guida di Silvestro, viene definito come "uomo antico." Il nonno di Silvestro è visto come "un guerriero antico." La cupola della chiesa nel paese di sua madre viene riconosciuta "antica nella memoria." Frequentissimi sono gli esempi dei termini "antico" e della sua variante "vecchio" in CS, tutti e due segni dell'integro carattere della antichità illesa dalle offese recate al mondo. Ed è a quest'integrità che il protagonista nella sua ricerca del "cuore puro" si vuole legare.

L'uomo è reso piú uomo non solo dal suo dolore ma anche dall'antichità delle sue pene. Questa è una delle cose che Silvestro impara nella progressione della sua coscienza al di là del regno della certezza illusoria dell'infanzia, al di là delle illusioni pure nelle cave di Colombo. Uomini come Calogero, Ezechiele e Porfirio, fanno solo parte della staticità storica della sofferenza umana resa inerte ed inerme dall'illusorio conforto del vino spremuto "per generazioni e generazioni" nelle "caverne dei secoli" nel sepolcrale "sottoterra del vino." Questo, Silvestro lo impara solo dopo il vivo contatto con la perpetua ripetizione del dolore nel regno sotterraneo della miseria del "genere umano operaio." $\grave{E}$ significativo il fatto che Silvestro scenda negli inferi della propria coscienza per assistere allo spettacolo del dolore collettivo.

La cognizione del dolore deve per forza esser preceduta dalla coscienza del male che il pellegrino si forma nell'incontro con gli agenti fascisti e con il Gran Lombardo sul treno. In questa tappa del suo sviluppo Silvestro impara a distinguere che la "puzza" attribuita a Coi Baffi e Senza Baffi appartiene ad una categoria del tutto etico-morale. A quel punto egli era ancora in preda ad astratti furori e anche a "vecchi significati," che lo rendevano insensibile al tipo di puzza che i suoi co-passeggeri riuscivano and intuire. La parola "puzza" viene ripetuta sedici volte nello spazio concentrato di tre pagine. Quest'intensità ripetitiva indica chiaramente lo scontro ideologico tra "puzza" e "nuovi doveri," "nuova e fresca coscienza," la lezione che gli viene impartita dal Gran Lombardo.

"Puzza" viene applicata due volte anche al simbolico cinese. La sua puzza, però, è quella degli offesi non degli offensori. L'idea di puzza viene ripetuta nella cantina di Colombo sotto 
forma di "tanfo." Questa è una terza categoria di puzza, cioè l'odore che deriva dalla coscienza che pute, non quello che emana da una misera esistenza. Il tanfo che si sente nella cantina di Colombo è l'inizio della putrefazione di una coscienza inerte. Silvestro fa appena in tempo ad uscire da questo stadio, che, come ben sa (si veda la ripetizione della frase "capo chino" nella sezione del vino, un'espressione che si riscontra all'inizio del viaggio, nella tappa degli astratti furori) è un vicolo cieco nel labirinto dell'essere. È non vita, non essere, "la quiete nella non speranza." La vera via della salvezza è quella interna, la voce che gli parla dalla coscienza, il vero significato dell'esclamazione "Ehm!" pronunciata ripetutamente dal fratello Liborio.

Prima di concludere con l'ultima fase del viaggio, vale a dire l'ascesi o l'integrazione, bisogna analizzare l'uso della ripetizione nella sfera della quarta dimensione per la quale Silvestro viene preparato dall'incontro con la madre. Questa tappa rappresenta la rigenerazione e la rinascita simbolica del pellegrino. Ed è anche a questo punto che acquistano nuovi significati le comunissime parole "donna" e "uomo."

Prima di arrivare da Concezione, Silvestro si trovava munito da un entusiasmo ispiratogli dall'odore delle carrube che aveva assorbito nella locanda, e dal sapore del formaggio sul traghetto. In piú, aveva imparato un nuovo significato della parola "puzza," e aveva anche ascoltato il messaggio dei "nuovi doveri" e della "nuova e fresca coscienza" dal Gran Lombardo. Tuttavia, era ancora in preda ad astratti furori, che si trasformano questa volta nella ripetizione della indifferenza che sentiva. Tracciando le varie fasi della sua indifferenza si vedrà non solo l'uso simmetrico e programmatico della ripetizione vittoriniana, bensí la progressiva scomparsa degli astratti furori man mano che le idee e le cose cominciano a materializzarsi una volta che si avvincina al "cuore puro" e alla quarta dimensione.

Al capitolo ottavo, la parola "Siracusa" viene ripetuta dieci volte il che, simulando il ritmo del treno che si avvicina a destinazione, mette a fuoco non solo la presenza fisica di Siracusa, ma anche l'immagine di essa nell'animo di Silvestro. Allo stesso modo, la parola "montagne," ripetuta ottantuno volte nel romanzo (le montagne sono generalmente simbolica rappresentazione di permanenza, integrità e solidità), a distanza e a contatto, specialmente quando il protagonista sta per arrivare dalla madre, serve a suggerire l'ascesi fisica e l'àncora psicologica che Silvestro invoca nella sua indifferenza. Viste prima come com- 
ponenti astratte del paesaggio onirico, le montagne si materializzano, acquistando forma e significato nella graduale transizione dal non essere all'essere.

La frase "Ma guarda, sono da mia madre!" è ripetuta una volta a pagina 599, e un'altra volta alla pagina seguente del decimo capitolo. La distanza che separa le identiche esclamazioni viene colmata dalla modulazione grammaticale ed esistenziale del verbo essere:

E mi parve che essere là non mi fosse indifferente, e fui contento di esserci venuto, non essere rimasto a Siracusa. . . Questo era il piú importante nell'essere là ... e lo trovavo improvviso, esserci . . . e credevo di essere entrato a viaggiare in una quarta dimensione. Pareva che non ci fosse stato nulla . . . tra l'essere a Siracusa e l'essere là e che essere là fosse effetto della mia decisione d'un movimento della mia memoria, non del mio corpo, [questo è l'indice piú preciso del viaggio interno] e cosí anche il mattino nell'essere là ... e il piacere di esserci; e nemmeno provavo rammarico per non aver potuto esserci la sera prima . . . come se quella luce fosse ancora del giorno 8 e non del giorno 9, o fosse d'un giorno in una quarta dimensione. (CS, p. 600)

Le diverse voci del verbo essere mettono in chiaro rilievo il Dasein del protagonista, tanto diverso dalla litania del nulla del primo capitolo. Nella quarta dimensione - convergenza di presente, passato, memoria e fantasia - la coscienza di Silvestro si innesta al sentimento del "due volte reale" e dello "in piú d'ora" producendo un "esserci" simultaneo del bambino e dell'uomo Silvestro.

L'indifferenza, di fronte a queste possibilità, si dirada come la nebbia al sole della Sicilia: "E mi parve ch'essere là non mi fosse indifferente" (p. 599); o dalla madre: "Riconobbi la soglia e non mi era indifferente di esserci" (p. 601) e, finalmente, quando annusa l'aringa arrostita: "non mi era indifferente" (p. 602). Dall'apatia all'entusiasmo, dall'astratto al concreto, dalla morte alla vita, insomma. La critica ha ben notato che CS è una fuga sulla parola "uomo." La parola "donna" ha anche una notevole frequenza nel romanzo. Tramite la ripetizione, l'essenzialità della donna viene messa a fuoco. La donna, una parola ripetuta piú di trenta volte in CS, viene esaminata da varie prospettive che accentuano la sua miseria, la sua gentilezza (la ricorrenza della parola "mani" conferisce maggiore rilievo a quest'ultimo particolare), la sua civetteria, il suo amore, e, nel caso di Concezione, il suo essere "piú donna," idea ribadita ed amplificata dalla statua 
di bronzo della "bella donna giovane nelle sue dimensioni due volte il naturale" (p. 706).

Allo stesso modo, ripetendo innumerevoli volte la parola "uomo," Silvestro, nella sua meditazione, sta penetrando l'essenza del significato della parola, che nella quarta dimensione si innalza ed attinge la vetta del nuovo significato nella frase "piú uomo." Con ogni ripetizione della parola, si rinnova e si riconferma l'urgenza del messaggio del "piú uomo" vittoriniano. "Uomo," come "montagne," nella ripetizione 'mantrica' acquista forma e significato nella coscienza del pellegrino.

A casa di Concezione viene raggiunta un'altra tappa interna del viaggio nella quarta dimensione. Questo si realizza tramite una serie particolare di ripetizioni verbali. I verbi conoscere, ricordare, vedere e pensare, appaiono simultaneamente nella sezione delle conversazioni di Silvestro con la madre, come ancelle della nascitura quarta dimensione. "Conoscere" viene ripetuto ventuno volte. La particella "ri," aggiunta al verbo conoscere serve pure a facilitare il varco ad una piú alta cognizione. "Ricordare" appare trenta volte partorito dal "riconoscere." "Vedere," che scaturisce dagli altri due verbi, è ripetuto cinquanta volte per segnare la vera e propria entrata nella quarta dimensione, una volta che si è sciolta la nebbia degli astratti furori. "Pensare," verbo appartenente alla categoria intellettiva ed anche psichica, ricorre piú di venti volte nel romanzo, e, come gli altri verbi menzionati, con maggiore concentrazione nella seconda parte del libro.

Una concordanza di CS dimostrerebbe sí una certa povertà linguistica, ma non una carenza di suggestività poetica. La ripetizione in Vittorini è indicazione evidente che l'autore ha qualche cosa da comunicare. Ripetendo le stesse parole, gli stessi motivi, temi, immagini continuamente, Vittorini ce li imprime nella coscienza dove silenziosamente generano nuovi significati. Questo tipo di iterazione, come nota Siciliano, "rivela una arcaicizzante teoria del linguaggio, per cui la parola è la cosa: - e la cosa viene offerta e riofferta al lettore, col pronunciarla in diverse variazioni sintattiche. ${ }^{\prime 8}$

Gilles Deleuze, autore di uno dei pochissimi studi monografici sulla ripetizione, cita Pius Servien nei cui libri, Principes d'esthetique e Science et poesie, si distingue tra due forme di linguaggio: "le language des sciences dominé par le symbole d'égalité, et ou chaque terme peut-être remplacé par d'autres; le langage lyrique, dont chaque terme, irremplaçable, ne peut-être que répété." 
Deleuze è dell'opinione che si può parlare di ripetizione solo quando si tratta di due fattori aventi "absolument le même concept." Allude poi alla forza evocatrice della ripetizione quando asserisce: "Mais de ces éléments discrets, de ces objets répétés, nous devons distinguer un sujet secret qui se répète à travers eux, véritable sujet de la répétition."

La coscienza di Silvestro è in formazione, cioè durante il viaggio egli accumula informazioni per poter riformare il suo stato d'animo amorfo. Ripetizione vuol dire rigenerazione per lui. La ripetizione a contatto, cioè concentrata, nella seconda parte del libro, condensa il significato allargandone la sfera di significazione e di senso recondito, come un "mantra." Nella ripetuta insistenza su verbi come "conoscere," "ricordare," "pensare," ecc., Silvestro sembra attingere a nuovi piani di dilatazione della coscienza. Tale procedimento lo aiuta anche a localizzare la posizione della evanescente quarta dimensione, nelle cui sfere egli acquista una conoscenza più acuta del "due volte reale." Ed è qui, come osserva Briosi, che "si accentuano i caratteri di astrattezza e di allusività della narrazione, e le parole, le battute del dialogo si succedono in un'atmosfera sempre piú rarefatta, capace di significati tanto piú 'essenziali' quanto piú sfuggenti, intraducibili in termini razionali." 10

La sofferenza che per Silvestro diventa realtà "due volte reale," per Ezechiele scrivano, resta formula retorica vuota. II "mondo offeso" salmodiato da Ezechiele diventa semplice litania non capace di trascendere la barriera linguistica. La ripetizione della frase non libera lui e i suoi amici dalla disperazione e dalla consolazione del vino. In tutto, la parola "offeso" e le sue varianti vengono ripetute quarantaquattro volte cristallizzandosi nel nonsignificato. Lo stesso vale per la ripetizione di "soffrire" e di "vino," che ricorrono trentadue e trentacinque volte rispettivamente. Anche l' "acqua viva," ripetuta diciassette volte sembra essere sopraffatta dall'inerzia del vino.

"Ehm!" vera voce della coscienza, è ripetuto venti volte nelle ultime pagine di CS. È a questo punto che il pellegrino si reintegra nell'ordine del dolore terrestre e cosmico. "Ehm!"' è il messaggio di solidarietà che gli proviene dal sottoterra, dalla morte, nel cimitero dove il dolore acquista dimensioni storiche ed universali. Ed è tramite questa parolina che Silvestro comunica il significato del dolore ai piccoli siciliani che per la prima volta chiedono: "Ed è molto soffrire?" 
Nel viaggio interno di CS non si arriva all'illuminazione nel senso mistico, bensí alla cognizione del dolore, allo stesso tempo cosmico e terrestre. Silvestro non trascende la realtà della sofferenza umana, come non la trascende Vittorini sublimandola nella torre eburnea della poesia. Il messaggio di Vittorini resta tutto umano (come lo è anche il suo concetto di Cristo): rendersi conto della sofferenza e dell'offesa e tornare a operare nella realtà munito di un sentimento di "nuovi ed altri doveri" e di una "nuova e fresca coscienza."

Le ripetizioni di Vittorini generano quella magia di cui parla nella sua prefazione al Garofano rosso, che è fede nel potere che ha la parola nel trasformare la sostanza di una cosa coll'impegno ulteriore di non "lasciare che la verità appaia morta," di afferrare la realtà "come insieme anche di parti e di elementi in via di formazione."11

\section{Università di Toronto \\ Scarborough College}

\section{NOTE}

1 Heinrich Lausberg, Elementi di retorica (Bologna: Società editrice Il Mulino, 1969). Oltre all'analisi di varie tecniche di ripetizione tali la "geminatio" (ripetizione a contatto), la "duplicatio" (ripetizione a distanza), la "gradatio," l'anafora, il polyptoton ecc., nella letteratura classica, risulta chiaro dallo studio del Lausberg che una funzione principale della ripetizione è di creare un "amplificatio" di emozioni che ci permette di arrestare il flusso continuo di informazioni offrendoci "il tempo di 'gustare' emozionalmente il contenuto dell'informazione che viene appunto accentuato e posto in evidenza per l'importanza che deve assumere" (p. 132). Questa stessa funzione viene anche messa in rilievo da Gilles Deleuze il quale afferma che "la répétition ne change rien dans l'objet qui se répète, mais elle change quelques chose dans l'esprit qui la contemple" : Différence et répétition (Paris: Presses de l'université de France, 1968), p. 33.

2 La parola "mantra," che di recente è apparsa anche nei dizionari inglesi e americani, significa letteralmente "veicolo del pensiero," cioè, benché il vocabolo "mantra" non esprima nessun significato specifico, il semplice suono emesso diventa strumento o mezzo che facilita l'accesso a significati reconditi della coscienza tramite la ripetizione. Il "mantra" per eccellenza degli indú è la sillaba sacra "OM" che ha la forza di risvegliare profonde cognizioni di verità nello spirito umano. Il concetto della potenza dei suoni, secondo lo studioso inglese John Blofeld, non è totalmente assente nella nostra tradizione giudeo-cristiana. È ancora riscontrabile in parole-suoni come "Amen" e "Alleluia" che, secondo Blofeld non vengono tradotti per conservare il loro originario valore "mantrico." $\mathrm{Si}$ veda il suo Mantras: Sacred Words of Power (London: Allen \& Unwin, 1977), p. 85. 
Il "mantra" è una parola-suono la cui ripetizione continua ha il potere di sprigionare l'energia latente che è normalmente bloccata in varie zone, o plessi, del corpo umano. Nel sistema filosofico indiano queste zone vengono chiamate "chakra." Il "mantra" opera nei "chakra" e desta nello spirito un'intuitiva comprensione di verità altrimenti impossibile ad esprimere con parole. Il "mantra," dunque, nella tradizione mistica indú, è visto come un mezzo fisico-spirituale per facilitare l'ascesi intellettiva-coscienziale. Infatti, asserisce lo studioso Mircea Eliade, la ripetizione dei mantra annulla la realtà del mondo secolare (ciò che non fa mai Vittorini), un primo passo mentale indispensabile nell'acquisizione di realtà piú profonde: Le Yoga: Immortalité et liberté (Parigi: Librairie Payot, 1954) p. 216.

I due termini "mantra" e "chakra" vengono a combaciare nella progressione della coscienza nelle varie discipline orientali come lo yoga, il trantismo, il buddismo ecc. Agendo sui centri di energia latente (i "chakra" sono sempre raffigurati in forma di ruota che ci ricorda "la ruota" del viaggio in CS), i "mantra" causano una graduale ascesi dell' energia (la quale, chiamata "kundalini," viene simbolicamente rappresentata da un serpente dormiente) in forma di spirale, dal punto piú basso a quello piú alto del corpo. I "chakra," sette in tutto, sono disposti nell'ordine seguente nel corpo umano: il primo è alla base della spina dorsale, la zona della materia, l'inerzia, la nascita del suono e dell'olfatto; il secondo è situato nella zona della riproduzione sessuale maschile, luogo dell'elemento acqua, del respiro, e della categoria sensoriale del gusto; il terzo si trova nella zona lombare ed è messo in rapporto all'elemento fuoco e alla vista; il quarto, situato nella zona del cuore (plesso solare), corrisponde all'elemento aria, al tatto e al fallo; il quinto, nella gola, corrisponde all'etere e all'udito; il sesto, posto fra le sopracciglia, è la sede delle facoltà intellettive in cui confluiscono i primi cinque "chakra"; il settimo, trovandosi al di sopra del cranio, non fa parte del corpo in quanto costituisce lo stadio finale dell'integrazione cosmica totale, cioè "nirvana" (Eliade, 1954, pp. 241-44). Oltre alle corrispondenze esoteriche elencate da Eliade servirebbe anche ricordarci della funzione biochimica specifica delle varie glandole associate ai diversi punti vitali del corpo umano: organi riproduttivi, surrenale, timo, tiroide e glandola pituitaria.

In questo studio faremo solamente uso riferenziale dei termini filosofici indú quali "mantra," "chakra," nirvana, e cosí via, a scopo di semplificare il dinamismo interno e la struttura esterna della ripetizione in CS attingendo a delle tecniche già sperimentate nella ricerca della verità, e non per proporre un paragone con l'uso che ne fa Vittorini.

3 Sono ormai ben note le dichiarazioni che Vittorini fece nel 1948 sul tema dello scrittore e della verità: "noi si esercita con ogni libro nel ricominciare a dire la verità . . . ripeterla ogni giorno non in qualche altra sua consistenza ma in qualche altro suo aspetto che la varia, che la rinnova. . . . Si tratta di non lasciare che la verità appaia morta. Essa è presente tra noi per la continuità delle nostre correzioni, delle nostre aggiunte, delle nostre ripetizioni . . .": Elio Vittorini: Le opere narrative, a cura di Maria Corti (Milano: Mondadori, 1974), pp. 428-29. Tutte le citazioni di Conversazione in Sicilia si riferiranno a questa edizione e verranno segnate dal numero della pagina direttamente nel testo.

4 Giovanni Cecchetti, "Elio Vittorini," Italica, 29 (Marzo 1952), 7.

5 Bruce Kavin, Telling it Again and Again: Repetition in Literature and Film (Ithaca: Cornell University Press, 1972) p. 39.

6 E.K. Brown, Rhythm in the Novel (Toronto: University of Toronto Press, 1950). Il "simbolo in espansione" ("expanding symbol") è un tipo di "amplificatio," ovvero una progressiva rivelazione dalla parte di un autore il quale, conscio di non poterci offrire tutto in una volta il nocciolo del suo significato, si limita a svelarcelo in una graduale sequenza d'intuizioni. Questo gli risulta possibile 
tramite l'uso della ripetizione bilanciata dalla variazione attraverso la quale una parola o un concetto ripetuto in diversi contesti acquista significato ed autonomia.

7 Si veda il nostro articolo "Vittorini's Synaesthetic Use of Acoustic Imagery" in Canadian Journal of Italian Studies, V, nn. 1-2 (Fall-Winter 1981-1982).

8 Enzo Siciliano, Prima della poesia (Firenze: Vallecchi, 1965), p. 22.

9 Deleuze, Différence et répétition, p. 8.

10 Sandro Briosi, Invito alla lettura di Vittorini (Firenze: La Nuova Italia, Il Castoro, 1970), p. 63.

11 Vittorini, "Prefazione alla prima edizione del Garofano Rosso," in Opere narrative I, p. 429. 